\title{
AFECTO POSITIVO COMO INDICADOR DE LAS ATRIBUCIONES ACADÉMICAS EN MATEMÁTICAS
}

\author{
Ricardo Sanmartin López \\ Universidad de Alicante, España. - ricardo.sanmartin@ua.es \\ María E. Urrea-Solano \\ Universidad de Alicante, España. \\ María J. Hernández-Amorós \\ Universidad de Alicante, España. \\ Lucía Granados Alós \\ Universidad Internacional de Valencia, España. \\ Nelly G. Lagos San Martín \\ Universidad del Bío-Bío, Chile. \\ José Manuel García Fernández \\ Universidad de Alicante, España \\ https://doi.org/10.17060/ijodaep.2017.n1.v3.971
}

Fecha de Recepción: 3 Febrero 2017

Fecha de Admisión: 1 Abril 2017

\section{RESUMEN:}

El objetivo del presente estudio fue el de analizar las diferencias de las puntuaciones en las atribuciones académicas en Matemáticas (éxito y fracaso atribuido a la capacidad, esfuerzo y causas externas) de estudiantes españoles de Educación Primaria en función de altas y bajas puntuaciones de afecto positivo. Para conseguir este objetivo, se recurrió a una muestra 962 de estudiantes de Educación Primaria (8-11 años de edad). Para obtener la medición de las variables analizadas, se utilizó la subescala de afecto positivo del Positive and Negative Affect Schedule for Children (PANAS) y la subescala de Matemáticas de la Sydney Attribution Scale (SAS). Tras llevar a cabo la prueba de Levene, se empleó la prueba $t$ de Student para analizar la existencia de diferencias estadísticamente significativas en las puntuaciones de atribuciones académicas entre los grupos de altos y bajos niveles de afecto positivo. En este sentido, se descubrió que los alumnos con altas puntuaciones de afecto positivo atribuyeron más sus éxitos y fracasos en Matemáticas a causas externas. Por el contrario, los estudiantes con bajos niveles de afecto positivo atribuyeron sus fracasos a la falta de capacidad y esfuerzo. Los resultados del presente trabajo amplían el conocimiento del afecto en la infancia y posibilitan el trabajo futuro de mejora en estos aspectos.

Palabras clave: afecto positivo; atribuciones académicas; Matemáticas; PANAS; Educación Primaria. 


\title{
AFECTO POSITIVO COMO INDICADOR DE LAS ATRIBUCIONES ACADÉMICAS EN MATEMÁTICAS
}

\begin{abstract}
:
Positive affect as an indicator of the academic attributions in Mathematics

The aim of the current study was to analyse the differences in the scores of academic attributions in Mathematics (success and failure attributed to capacity, effort and external causes) of Spanish students of primary education according to high and low scores in positive affect. To fulfil this target, a sample of 862 students of primary education (8-11 years old) was recruited. To obtain the scores of the analysed variables, the positive affect subscale of the Positive and Negative Affect Schedule for Children (PANAS) and the Mathematics subscale of the Sydney Attribution Scale (SAS) were used. After performing the Levene's test, the Student's t-test was used to analyse the existence of statistically significant differences in the scores of academic attributions between students with high scores in positive affect and students with low scores. In this sense, it was proved that students with high marks in positive affect attributed more their failures and successes in Mathematics to external causes. On the contrary, students with low levels of positive affect attributed more their failures to the lack of capacity or effort. The presented results broad the knowledge of affect during childhood and possibility the future work in improving these aspects.
\end{abstract}

Keywords: positive affect; academic attributions; Mathematics; PANAS; primary education.

Desde que Watson, Clark y Tellegen (1988) establecieron la división de las dimensiones afectivas de una persona en dos categorías independientes entre sí, afecto positivo (AP) y afecto negativo (AN), la literatura científica ha realizado uso de la misma para analizar variables psicológicas y emocionales que intervienen en el desarrollo de los individuos.

En este sentido, diversos autores han llevado a cabo estudios para analizar las dimensiones afectivas con variables como el estrés, la ansiedad, la autoestima, el optimismo 0 el bienestar, entre otros (Di Fabio y Bucci, 2015; Garcia, Nima y Kjell, 2014; Sandín, 2003). A raíz de las investigaciones comentadas, la dimensión de AP ha sido relacionada con altas puntuaciones en dimensiones adaptativas como pueden ser la autoestima, el optimismo 0 el bienestar personal. Por lo que puede vislumbrarse la asociación del AP con dimensiones positivas y adaptativas del desarrollo eficaz de las personas.

A pesar de que es bastante elevado el número de asociaciones entre el afecto y las variables psicológicas y emocionales de los individuos, es cierto que la relación entre la dimensión afectiva y elementos intervinientes en el contexto escolar se ha estudiado en menor medida. En esta línea se pueden encontrar estudios como el de Linnenbrink y Pintrich (2004) que establecen la relación entre el afecto y los procesos cognitivos que tienen lugar en los ambientes académicos. No obstante, existe una dimensión importante dentro del campo de las características académicas cuyo análisis puede aportar información importante al campo de estudio de las características afectivas de las personas, dicha dimensión se conoce como las atribuciones académicas propuestas por Weiner (1986).

Las atribuciones académicas hacen referencia a la justificación que establece un individuo para indicar la causa de sus aciertos y sus errores. Teniendo en cuenta las palabras de Weiner (1986), los indicativos que se suelen situar como causas de los éxitos y los fracasos son la capacidad, el esfuerzo, la suerte y la dificultad de una tarea. Teniendo en cuenta esta idea, se puede identificar la posible potencialidad que puede tener el análisis de dichas dimensiones según las dimensiones afectivas, ya que los resultados académicos de los estudiantes tienen una importancia muy grande en sus estados afectivos.

Por consiguiente, se ha podido observar en el estudio de Sanmartín, Inglés, Vicent, Gonzálvez 
y García-Fernández (2016) como se trata de evaluar la diferencia en las puntuaciones obtenidas en atribuciones académicas en Lengua en función del AP. En dicho trabajo se concluyó que altas puntuaciones de AP tendían a atribuir más el éxito y el fracaso a causas externas, mientras que bajas puntuaciones en AP atribuían sus fracasos a la capacidad y el esfuerzo. Estos resultados abren la posibilidad a la relación que se ha comentado anteriormente acerca de la incidencia que tienen los éxitos y los fracasos en la dimensión afectiva de un individuo.

A pesar de haber estudiado la relación entre atribuciones académicas y afecto en el área de Lengua, existe un área en los colegios que también merece ser estudiada, el área de Matemáticas. Pérez-Tyteca, Monje y Castro (2013) comentan en su estudio que los estudiantes que experimentan ansiedad en las respuestas matemáticas y sentimientos negativos hacia ellas tienen peores resultados, presentan una baja capacidad percibida para enfrentarse a la asignatura y muestran inseguridad, respecto a sus compañeros sin ansiedad. Gómez-Chacón (2010) realiza un análisis de los diversos estudios que han estudiado la relación entre afecto y Matemáticas y llega a la conclusión que el estudio de las creencias que hay instauradas en el alumnado podría ser un tema interesante de estudio. A su vez, también se indica la necesidad de estudiar la relación del afecto con componentes específicos del aprendizaje (Gómez-Chacón, 2002). Por último, Gil, Lorenzo y Guerrero (2005) establecen que la aparición de actitudes negativas de características ambientales y personales hacia las Matemáticas podrían ser los desencadenantes de los fracasos de los resultados en esta área, por lo que su estudio en las primeras etapas educativas es muy interesante.

\section{EL PRESENTE ESTUDIO}

Por tanto, tras la revisión bibliográfica realizada, se ha podido constatar la necesidad de seguir estudiando la relación entre el afecto y variables relacionadas con el desempeño en Matemáticas y la importancia de añadir nuevas investigaciones al estudio del afecto debido al bajo número de investigaciones que analizan la dimensión afectiva en la etapa de la infancia.

En consecuencia, el presente trabajo se plantea el análisis de las diferencias en las puntuaciones medias de las dimensiones de las atribuciones académicas de Matemáticas (Éxito y fracaso atribuidos a capacidad, esfuerzo o factores externos) entre los individuos con altos y bajos niveles de AP en la etapa de Educación Primaria. En este estudio se ha elegido el AP debido a que es una dimensión que promueve una imagen positiva del alumno y unas características emocionales adaptativas. En este sentido, se espera que:

Hipótesis 1: Ios participantes con altos niveles de AP tenderán a atribuir el éxito y el fracaso en Matemáticas a causas externas (Pérez-Tyteca et al., 2013; Sanmartín et al., 2016).

Hipótesis 2: Ios participantes con bajos niveles de AP tenderán a atribuir sus fracasos en el área de Matemáticas a la falta de capacidad y esfuerzo (Pérez-Tyteca et al., 2013; Sanmartín et al., 2016).

\section{MÉTODO}

\section{Participantes}

Los participantes de este estudio fueron elegidos por muestreo aleatorio por conglomerados. En consecuencia, el presente estudio contó con una muestra que estuvo compuesta por un total de 862 estudiantes de Educación Primaria, con edades comprendidas entre los 8 y los 11 años de edad ( $M$ $=9.52 ; D E=1.53)$. La totalidad de los participantes estaban cursando sus estudios en centros públicos y concertados en las provincias de Alicante y Murcia. Respecto al número de participantes en función de sexo, es importante establecer que en el estudio participaron 424 chicas y 438 chicos. Por lo que concierne a la división de los participantes por edad, un total de 241 estudiantes tenían 8 años, 241 estudiantes tenían 9 años, 198 tenían 10 años y 182 tenían 11 años. 


\section{AFECTO POSITIVO COMO INDICADOR DE LAS ATRIBUCIONES ACADÉMICAS EN MATEMÁTICAS}

\section{Medidas}

Los instrumentos de medida que se han utilizado en el presente estudio son los siguientes:

The 10-Item Positive and Negative Affect Schedule for Children (PANAS-C; Ebesutani et al., 2012). Esta escala es una medida de auto-informe con escala Likert de 5 puntos (1: "nunca" hasta 5: "siempre"). En ella se aportan dos subescalas para medir tanto el AP como el AN, con cinco ítems cada una. Para el presente trabajo se ha utilizado la subescala del AP que trata de utilizar cinco adjetivos positivos de afecto para valorar la frecuencia con que el sujeto los ha experimentado durante las últimas semanas. El coeficiente de consistencia interna de la subescala en esta investigación fue adecuado $(\alpha=.81)$.

The Sydney Attribution Scale (SAS; Marsh, Cairns, Relich, Barnes y Debus, 1984; validada y traducida al español por Núñez y González-Pienda, 1994). Este instrumento proporciona un total de 24 situaciones hipotéticas que los participantes deben responder a través de una escala de respuesta tipo Likert de 5 puntos, en la que el 1 se considera falso y el 5 verdadero. Para la aplicación del presente estudio, cabe mencionar que solamente se emplearon las situaciones que hacían referencia al área de Matemáticas. Los índices de consistencia interna para todos los elementos del área de Matemáticas (éxito y fracaso en función de esfuerzo, capacidad y causas externas) fueron adecuados, encontrándose en un margen de .70 a .84 .

\section{Procedimiento}

La obtención de datos de la presente investigación se realizó pidiendo, en primer lugar, el consentimiento firmado de participación de todos los padres o tutores legales de los alumnos. Posteriormente, cuando todos los participantes estaban dispuestos y legalmente habilitados para participar, se les comentó que la prueba era de cumplimentación voluntaria y anónima, para que de este modo contestaran de forma más sincera. La sesión de aplicación tuvo una duración de unos 20 minutos y un miembro del equipo de investigación estuvo presente para resolver cualquier duda durante la cumplimentación de los cuestionarios.

\section{Análisis estadísticos}

En primer lugar, el total de participantes se clasificó en dos grupos en base a sus puntuaciones en AP. Es decir, por un lado, se agruparon los estudiantes con puntuaciones altas en AP, aquellas puntuaciones que se encontraban en el centil 75 o superior. Por otro lado, los participantes que obtuvieron bajos niveles de AP se agruparon en otro colectivo, aquellos estudiantes cuyas puntuaciones se encontraban en el centil 25 o inferior. Posteriormente, se comprobó el supuesto de homogeneidad de varianzas mediante la prueba de Levene. Una vez confirmado, se comprobaron las diferencias en las puntuaciones en atribuciones académicas en Matemáticas de los dos colectivos (alto AP y bajo AP) mediante la prueba $t$ de Student para muestras independientes. Todos los cálculos estadísticos se realizaron con el paquete estadístico SPSS 22.

\section{RESULTADOS}

La Tabla 1 muestra los resultados de los análisis estadísticos descritos anteriormente. Como se puede observar, los participantes con altas puntuaciones en AP obtuvieron medias significativas más altas a la hora de atribuir el éxito y el fracaso a causas externas, en comparación con sus compañeros con bajas puntuaciones de AP. Por el contrario, los individuos con puntuaciones bajas en AP mostraron diferencias significativas a la hora de atribuir el fracaso más a la capacidad y al esfuerzo que sus compañeros con puntuaciones altas en AP. Respecto a la atribución del éxito a la capacidad y al esfuerzo no se encontraron diferencias estadísticamente significativas. 
Tabla 1

Prueba de Levene y prueba $t$ de Student para las seis dimensiones de atribuciones académicas en Matemáticas en función de altos y bajos niveles de AP

\begin{tabular}{lccccccccc}
\hline & \multicolumn{2}{l}{ Prueba de Levene } & \multicolumn{2}{l}{ Grupo Alto AP } & \multicolumn{2}{c}{ Grupo Bajo AP } & \multicolumn{2}{c}{ Sig. Estadística } \\
\hline SAS (Matemáticas) & $F$ & $p$ & $M$ & $D E$ & $M$ & $D E$ & $T_{169}$ & $p$ \\
\hline Capacidad (Éxito) & .24 & .619 & 2.8 & 1.32 & 2.75 & 1.25 & .68 & .492 \\
Esfuerzo (Éxito) & .13 & .715 & 3.34 & 1.11 & 3.15 & .98 & 1.12 & .261 \\
Causas externas (Éxito) & 1.71 & .192 & 3.91 & 1.10 & 3.58 & .97 & 2.01 & .046 \\
Capacidad (Fracaso) & .12 & .720 & 1.19 & 1.13 & 2.05 & 1.21 & -4.74 & $<.001$ \\
Esfuerzo (Fracaso) & 2.72 & .101 & 1.59 & 1.08 & 2.34 & .94 & -4.69 & $<.001$ \\
Causas externas (Fracaso) & 1.88 & .172 & 4.43 & .95 & 3.88 & .86 & 3.84 & $<.001$ \\
\hline
\end{tabular}

\section{DISCUSIÓN}

El pretexto y el objetivo del presente estudio fue analizar la existencia de diferencias estadísticamente significativas en las puntuaciones de atribuciones académicas en Matemáticas en un grupo de alumnos de Educación Primaria en función de las puntuaciones de AP.

En primer lugar, teniendo en cuenta los resultados obtenidos en la investigación se ha podido constatar que la Hipótesis 1 ha sido aceptada, ya que los alumnos con altas puntuaciones en AP han obtenido puntuaciones significativamente más altas a la hora de atribuir sus éxitos y fracasos a causas externas. Los resultados obtenidos en esta dimensión coinciden con lo comentado por Sanmartín et al. (2016), aunque el área que se analizó en dicha investigación fue el área de Lengua. Teniendo en cuenta que ambas investigaciones han obtenido los mismos resultados, sería interesante tener en cuenta que cuando los participantes tienden a asociar sus éxitos y fracasos a causas externas tienden a relacionarse con altas puntuaciones en AP, lo que indicaría una protección de su imagen positiva personal. Estos resultados también estarían en la línea de los aportados por 0p't Eynde y De Corte (2003), ya que estos autores establecieron que a pesar de que los docentes trataban de infundir buenas actitudes hacia las matemáticas y creencias positivas, los estudiantes no lograban asimilarlas y no las incluían en su desarrollo afectivo. En este sentido, se puede comprobar como los alumnos con alto AP no asocian su creencia de éxito en las atribuciones a su capacidad o esfuerzo, sino que a elementos externos.

En segundo lugar, la Hipótesis 2 también ha sido confirmada, ya que los alumnos con bajas puntuaciones en AP han tendido a atribuir significativamente más sus fracasos a la falta de capacidad y esfuerzo. Como se ha comentado en la introducción, estos resultados coinciden con la investigación de Pérez-Tyteca et al. (2013) y Sanmartín et al. (2016), en el área de Lengua. A su vez, las investigaciones de Gonske (2002) e Isiksal, Curran, Koc y Askun (2009) establecen la aparición del elemento de percibir poca capacidad propia e inseguridad a la hora de resolver actividades Matemáticas cuando han experimentado ansiedad ante la asignatura. En el caso que se presenta en la actual investigación, cabe mencionar que alumnos con niveles bajos de AP pueden mostrar una 


\section{AFECTO POSITIVO COMO INDICADOR DE LAS ATRIBUCIONES ACADÉMICAS EN MATEMÁTICAS}

imagen afectiva más afectada debido a que han atribuido sus fracasos a la falta de capacidad y esfuerzo, lo que genera una inseguridad a la hora de enfrentarse a actividades del área de Matemáticas (Guerrero, Nieto y Gil, 2005).

A pesar de los puntos positivos del estudio que se presenta, es importante mencionar que la investigación posee una serie de limitaciones. Primeramente, debido a que los análisis estadísticos utilizados indican la existencia de una relación entre las variables pero no se puede establecer una relación causal entre las mismas, es importante que en el futuro se lleven a cabo estudios longitudinales para analizar la posible relación casuística. Por otro lado, también es importante tener en cuenta en el futuro variables como el sexo, la edad o incluso la dimensión del AN para complementar los resultados obtenidos en el presente trabajo. Por lo que respecta a la recogida de datos, PérezTyteca et al. (2013) establecen la necesidad de utilizar herramientas cualitativas para conocer las causas que pueden justificar la ansiedad hacia las matemáticas y la autoconfianza en la vida académica de los estudiantes. En este sentido, ampliar con metodología cualitativa 0 incluso teniendo en cuenta la visión de los docentes o las familias podrían aportar nuevas formas de entender la relación detectada. Por último, en un futuro se podría relacionar las atribuciones en matemáticas con otras dimensiones que también han sido relacionadas con el afecto y que tienen importancia en el desarrollo académico de los estudiantes, como puede ser el rechazo escolar y el perfeccionismo, entre otros (Gonzálvez et al., 2016; Inglés, García-Fernández, Vicent, Gonzálvez y Sanmartín, 2016; Inglés, Gonzávez, García-Fernández, Vicent y Sanmartín, 2016; Vicent, Gonzálvez, Sanmartín, García-Fernández e Inglés, 2016).

En definitiva, a pesar de las limitaciones señaladas, los resultados del presente estudio aportan información importante y necesaria al campo de estudio del afecto y las atribuciones académicas en Matemáticas, porque señala diferencias encontradas en función de los niveles de afecto en una etapa que no ha sido investigada en profundidad como es la primera infancia. Se espera que en el futuro se siga ampliando el conocimiento de la relación de ambas variables con futuras investigaciones.

\section{CONCLUSIONES}

Las principales conclusiones del presente trabajo son, por un lado, la identificación de que los alumnos con altos niveles de AP tienden a atribuir sus éxitos y fracasos en Matemáticas a factores externos. Por otro lado, los estudiantes con bajos niveles de AP tienden a atribuir sus fracasos en Matemáticas a la capacidad y al esfuerzo. Es importante los resultados obtenidos, ya que es necesario desde los centros educativos revertir la situación de que los alumnos a la hora de enfrentarse a las Matemáticas no confíen en sus capacidades y esfuerzos para superar los problemas que se planteen. De esta forma, los alumnos desarrollarán actitudes de control y adaptativas en el área de Matemáticas.

\section{REFERENCIAS BIBLIOGRÁFICAS}

Di Fabio, A. y Bucci, 0. (2015). Affective profiles in Italian high school students: Life satisfaction, psychological well-being, self-esteem, and optimism. Frontiers in Psychology, 6, 1-6. doi:10.3389/fpsyg.2015.01310

Ebesutani, C., Regan, J., Smith, A., Reise, S., Higa-McMillan, C. y Chorpita, B. F. (2012). The 10-item positive and negative affect schedule for children, child and parent shortened versions: Application of item response theory for more efficient assessment. Journal of Psychopathology and Behavioral Assessment, 34(2), 191-203. http://dx.doi.org/10.1007/s10862-011-9273-2

Garcia, D., Nima, A. A. y Kjell, 0. N. (2014). The affective profiles, psychological well-being, and harmony: Environmental mastery and self-acceptance predict the sense of a harmonious life. PeerJ, 2(e259), 1-21. doi:10.7717/peerj.259 
Gil, N., Lorenzo, J.B. y Guerrero, E. (2005). El dominio afectivo en el aprendizaje de las Matemáticas. Una revisión de sus descriptores básicos. Revista Iberoamericana de Educación Matemática, (2), 15-32. Disponible: < https://dialnet.unirioja.es/servlet/articulo?codigo=2218956> ISSN-e $1815-0640$.

Gómez-Chacón, I.Mạ (2002). Afecto y aprendizaje matemático: causas y consecuencias de la interacción emocional. En J. Carillo (Ed.), Reflexiones sobre el pasado, presente y futuro de las Matemáticas (pp. 197-227). Huelva: Universidad de Huelva.

Gómez-Chacón, I.Mํa . (2010). Tendencias actuales en investigación en matemáticas y afecto. En M.M. Moreno, A. Estrada, J. Carrillo y T.A. Sierra, (Eds.), Investigación en Educación Matemática XIV (pp. 121-140). Lleida: SEIEM.

Gonske, T. L. (2002). Relationships among mathematics anxiety, beliefs about the nature of mathematics and the learning of mathematics, and students learning approaches in non-traditional. (Tesis doctoral). Greeley: University of Northern Colorado.

Gonzálvez, C, Inglés, C.J., Kearney, C.A., Vicent, M., Sanmartín, R. y García-Fernández, J.M. (2016). School Refusal Assessment Scale-Revised: Factorial Invariance and Latent Means Differences across Gender and Age in Spanish Children. Frontiers in Psychology, 7, 2011. doi: 10.3389/fpsyg.2016.02011

Guerrero, E., Nieto, L. J. B. y Gil, N. (2005). El dominio afectivo en el aprendizaje de las Matemáticas. Una revisión de sus descriptores básicos. Unión: revista iberoamericana de educación matemática, (2), 15-32. Disponible: <https://dialnet.unirioja.es/servlet/articulo?codigo=2218956> ISSN 1815-0640.

Inglés, C.J., García-Fernández, J.M., Vicent, M., Gonzálvez, C. y Sanmartín, R. (2016). Profiles of Perfectionism and School Anxiety: A Review of the 2x2 Model of Dispositional Perfectionism in Child Population. Frontiers in Psychology, 7, 1403. doi: 10.3389/fpsyg.2016.01403

Inglés, C.J., Gonzálvez, C., García-Fernández, J.M., Vicent, M. y Sanmartín, R. (2016). Estudio correlacional entre el afecto negativo y el rechazo escolar. International Journal of Developmental and Educational Psychology. Revista INFAD de Psicología., 1(1), 95-102. Disponible: < http://rua.ua.es/dspace/handle/10045/53928>

Isiksal, M., Curran, J. M., Koc, Y. y Askun, C. S. (2009). Mathematics anxiety and mathematical selfconcept: considerations in preparing elementary-school teachers. Social Behavior and Personality, 37(5), 631-643. Disponible: < https://www.sbp-journal.com/index.php/sbp/article/view/1871>

Linnenbrink, E. A. y Pintrich, P. R. (2004). Role of affect in cognitive processing in academic contexts. En D. Y. Dai y R. J. Sternberg (Eds.), Motivation, emotion, and cognition: Integrative perspectives on intellectual functioning and development (pp. 57-87). Mahwah, NJ: Lawrence Erlbaum Associates, Inc.

Marsh, H. W., Cairns, L., Relich, J., Barnes, J. y Debus, R. L. (1984). The relationship between dimensions of self-attribution and dimensions of self-concept. Journal of Educational Psychology, 76, 3-32. http://dx.doi.org/10.1037/0022-0663.76.1.3

Núñez, J.C. y González-Pineda, J.A. (1994). Determinantes del rendimiento académico. Oviedo: Servicio de Publicaciones de la Universidad de Oviedo.

Op't Eynd, P. y De Corte, E (2003). Junior High students' mathematicsrelated beliefs systems: An empirical analisy of their internal and external structure. Padua: 10th Biennial Conference of the European Association for Research on Learning and Instruction.

Pérez-Tyteca, P., Monje, J. y Castro, E. (2013). Afecto y matemáticas. Diseño de una entrevista para acceder a los sentimientos de alumnos adolescentes. Avances de Investigación en Educación 


\section{AFECTO POSITIVO COMO INDICADOR DE LAS ATRIBUCIONES ACADÉMICAS EN MATEMÁTICAS}

Matemática, (4), 65-82. Disponible: < http://www.aiem.es/index.php/aiem/article/view/55>

Sandín, B. (2003). Escalas panas de afecto positivo y negativo para niños y adolescentes (PANASN). Revista De Psicopatología y Psicología Clínica, 8(2), 173-182. Disponible: < https://dialnet.unirioja.es/servlet/articulo?codigo=722206 > ISSN 1136-5420.

Sanmartín, R., Inglés, C. J., Vicent, M., Gonzálvez, C. y García-Fernández, J. M. (2016). Diferencias en las atribuciones académicas en Lengua en base al Afecto Positivo. International Journal of Developmental and Educational Psychology. Revista INFAD de Psicología., 2(1), 143-150. Disponible: < http://rua.ua.es/dspace/handle/10045/53929>

Vicent, M., Gonzálvez, C., Sanmartín, R., García-Fernández, J.M. e Inglés, C.J. (2016). Perfeccionismo socialmente prescrito y afecto en la infancia. International Journal of Developmental and Educational Psychology. Revista INFAD de Psicología., 1(1), 333-340. Disponible: < http://rua.ua.es/dspace/handle/10045/53968>

Watson, D., Clark, L. A. y Tellegen, A. (1988). Development and validation of brief measures of positive and negative affect: The PANAS scales. Journal of Personality and Social Psychology, 54(6), 1063-1070. Disponible: < http://psycnet.apa.org/index.cfm?fa=buy.optionToBuy\&id=198831508-001>

Weiner, B. (1986). An attributional theory of achievement motivation and emotion. New York: Springer-Verlag. 